\title{
Flavor diagonal tensor charges of the nucleon from $(2+1+1)$-flavor lattice QCD
}

\author{
Rajan Gupta, ${ }^{1, *}$ Boram Yoon, ${ }^{2, \dagger}$ Tanmoy Bhattacharya, ${ }^{1, \ddagger}$ Vincenzo Cirigliano, ${ }^{1, \S}$ \\ Yong-Chull Jang,,$\|$ and Huey-Wen Lin ${ }^{4,5, \pi}$
}

(PNDME Collaboration)

\author{
${ }^{1}$ Los Alamos National Laboratory, Theoretical Division T-2, Los Alamos, New Mexico 87545, USA \\ ${ }^{2}$ Los Alamos National Laboratory, CCS Division CCS-7, Los Alamos, New Mexico 87545, USA \\ ${ }^{3}$ Brookhaven National Laboratory, Upton, New York 87545, USA \\ ${ }^{4}$ Department of Physics and Astronomy, Michigan State University, East Lansing, Michigan 48824, USA \\ ${ }^{5}$ Department of Computational Mathematics, Science and Engineering, Michigan State University, \\ East Lansing, Michigan 48824, USA
}

(Received 4 September 2018; published 12 November 2018)

\begin{abstract}
We present state-of-the-art results for the matrix elements of flavor diagonal tensor operators within the nucleon state. The calculation of the dominant connected contribution is done using 11 ensembles of gauge configurations generated by the MILC Collaboration using the highly improved staggered quark action with $2+1+1$ dynamical flavors. The calculation of the disconnected contributions is done using seven (six) ensembles for the strange (light) quarks. These high-statistics simulations allowed us to address various systematic uncertainties. A simultaneous fit in the lattice spacing and the light-quark mass is used to extract the tensor charges in the continuum limit and at $M_{\pi}=135 \mathrm{MeV}$. Results for the proton in the $\overline{\mathrm{MS}}$ scheme at $2 \mathrm{GeV}$ are $g_{T}^{u}=0.784(28)(10), g_{T}^{d}=-0.204(11)(10)$ and $g_{T}^{s}=-0.0027(16)$. Implications of these results for constraining the quark electric dipole moments and their contributions to the neutron electric dipole moment are discussed.
\end{abstract}

DOI: 10.1103/PhysRevD.98.091501

\section{INTRODUCTION}

High-precision calculations of the matrix elements of flavor diagonal quark bilinear operators $\bar{q} \Gamma q$, where $\Gamma$ is one of the 16 Dirac matrices, within the nucleon state provide a quantitative understanding of a number of properties of nucleons and their interactions with electrically neutral probes. In this paper, we present results for the tensor charges $g_{T}^{u}, g_{T}^{d}$ and $g_{T}^{s}$ that give the contribution of the electric dipole moment (EDM) of these quark flavors to the EDM of the nucleon. They are defined as the nucleon matrix elements of the renormalized tensor operator

\footnotetext{
rajan@lanl.gov

†boram@lanl.gov

tanmoy@lanl.gov

§cirigliano@lanl.gov

"ypj@bnl.gov

hwlin@pa.msu.edu
}

Published by the American Physical Society under the terms of the Creative Commons Attribution 4.0 International license. Further distribution of this work must maintain attribution to the author(s) and the published article's title, journal citation, and DOI. Funded by SCOAP ${ }^{3}$.
$Z_{T} \bar{q} \sigma^{\mu \nu} q$ with $\sigma^{\mu \nu}=i\left[\gamma_{\mu}, \gamma_{\nu}\right] / 2, Z_{T}$ the renormalization constant and $q$ the bare quark field:

$$
\left\langle N(p, s)\left|Z_{T} \bar{q} \sigma_{\mu \nu} q\right| N(p, s)\right\rangle=g_{T}^{q} \bar{u}_{N}(p, s) \sigma_{\mu \nu} u_{N}(p, s)
$$

Experimentally, they can be extracted from semi-inclusive deep-inelastic scattering data [1-3]. These tensor charges also provide the hadronic input to the weakly interacting massive particle (WIMP)-nucleus cross section in dark matter models that generate tensor quark-WIMP operators [4].

New high-statistics data for both the connected and disconnected contributions to the tensor charges allow us to control the various systematic uncertainties and perform a chiral-continuum fit to obtain physical results. The lightquark disconnected contributions, which were neglected in our previous works [5,6], are $O(0.01)$; nevertheless, the data are precise enough to allow extrapolation to the continuum limit and $M_{\pi}=135 \mathrm{MeV}$. We also report a signal in the still smaller $g_{T}^{s}$, whose contribution to the neutron EDM can be enhanced versus $g_{T}^{u}$ by $m_{s} / m_{u} \approx 40$ in models in which the chirality flip is provided by the Standard Model Yukawa couplings. 
TABLE I. The number of configurations analyzed for the light $\left(N_{\text {conf }}^{l}\right)$ and strange $\left(N_{\text {conf }}^{s}\right)$ quarks, the corresponding number of random sources $\left(N_{\mathrm{src}}\right)$ sampled, and the ratio $N_{\mathrm{LP}} / N_{\mathrm{HP}}$ of low- to high-precision measurements made to estimate the disconnected quark loop contribution on each configuration.

\begin{tabular}{cccccc}
\hline \hline Ensemble & $N_{\text {conf }}^{l}$ & $N_{\text {src }}^{l}$ & $N_{\text {conf }}^{s}$ & $N_{\text {src }}^{s}$ & $N_{\mathrm{LP}} / N_{\mathrm{HP}}$ \\
\hline$a 15 m 310$ & 1917 & 2000 & 1919 & 2000 & 50 \\
$a 12 m 310$ & 1013 & 5000 & 1013 & 1500 & 30 \\
$a 12 m 220$ & 958 & 11000 & 958 & 4000 & 30 \\
$a 09 m 310$ & 1081 & 4000 & 1081 & 2000 & 30 \\
$a 09 m 220$ & 712 & 8000 & 847 & 10000 & $30 / 50$ \\
$a 09 m 130$ & & & 877 & 10000 & 50 \\
$a 06 m 310$ & 830 & 4000 & $200+340$ & $5000+10000$ & 50 \\
\hline \hline
\end{tabular}

\section{LATTICE METHODOLOGY}

All the calculations were done on ensembles with $2+1+1$ flavors of highly improved staggered quark (HISQ) fermions [7] generated by the MILC Collaboration [8].

In order to calculate the matrix elements of flavor diagonal operators, one needs to evaluate the contribution of both the "connected" and "disconnected" diagrams. The lattice methodology and our strategy for the calculation and analysis of the two-point and connected three-point functions using Wilson clover fermions on the HISQ ensembles has been described in Refs. [5,9-11] and for the disconnected contribution in Refs. [5,12].

The details of the calculation and analysis of the connected contributions on 11 ensembles covering the range $0.15-0.06 \mathrm{fm}$ in the lattice spacing, $M_{\pi}=$ $135-320 \mathrm{MeV}$ in the pion mass, and $M_{\pi} L=3.3-5.5$ in the lattice size have been presented in Ref. [13] and readers are referred to it. With these high-statistics data $\left[O\left(10^{5}\right)\right.$ measurements on $O(1000)$ configurations on each of the 11 ensembles], a chiral-continuum-finite-volume fit in the three variables $a, M_{\pi}^{2}$ and $M_{\pi} L$ was performed to control the systematic uncertainties due to lattice discretization, dependence on the quark mass and finite lattice size. The final results, in the $\overline{\mathrm{MS}}$ scheme at $2 \mathrm{GeV}$, for the connected contribution to the proton, are reproduced from Ref. [13]:
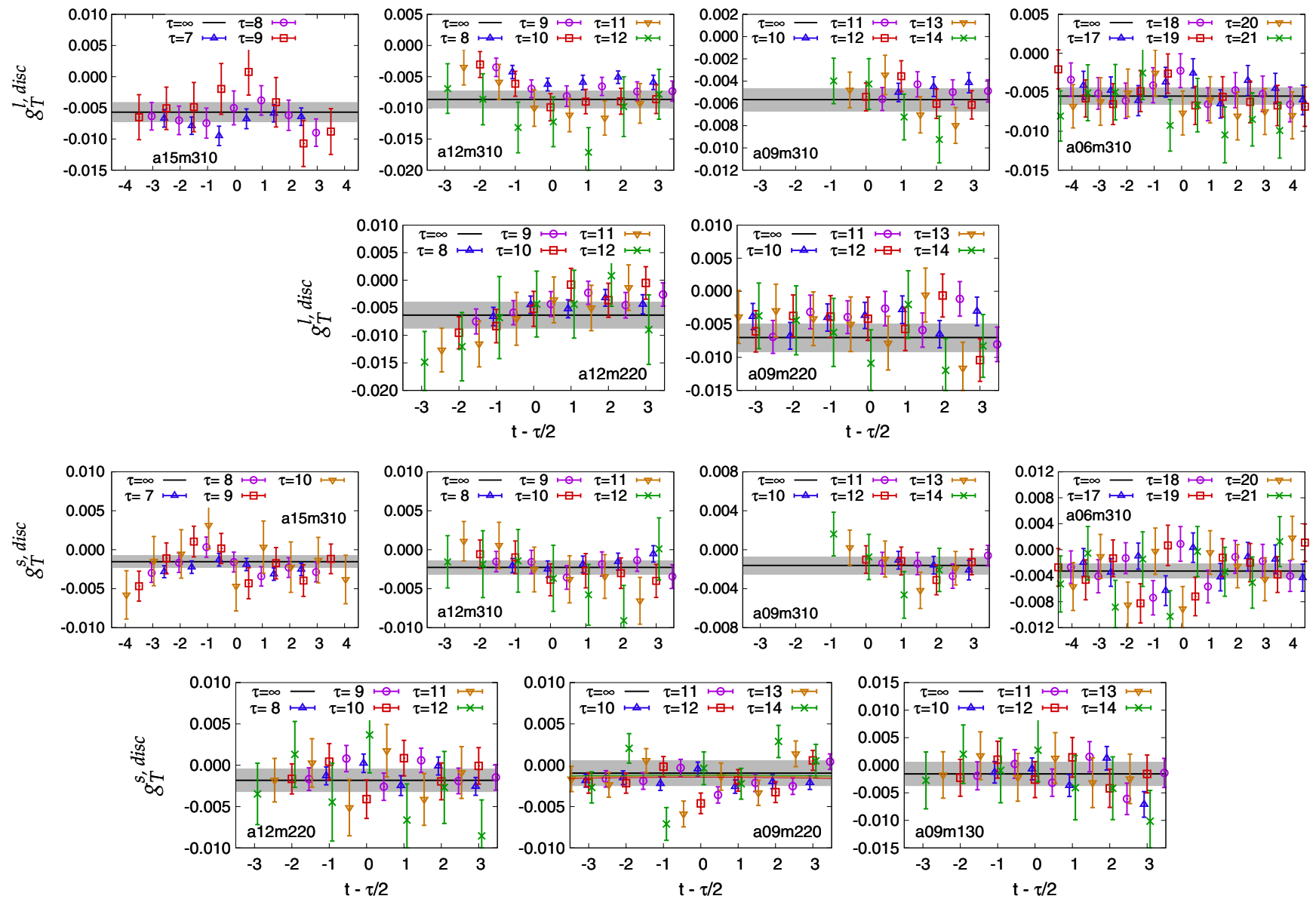

FIG. 1. The data for the unrenormalized disconnected contributions of the light $g_{T}^{l \text {,disc }}$ (top two rows) and strange $g_{T}^{s, \text { disc }}$ (bottom two rows) quarks. The ground state estimate is given by the solid black line within the gray band. It is obtained as the average over data at multiple $t$ and $\tau$ since no significant variation versus them is observed. 


$$
\begin{aligned}
g_{T}^{u-d} & =0.989(32), & & \left.g_{T}^{u+d}\right|_{\text {conn }}=0.590(25), \\
\left.g_{T}^{u}\right|_{\text {conn }} & =0.790(27), & & \left.g_{T}^{d}\right|_{\text {conn }}=-0.198(10) .
\end{aligned}
$$

In this paper, we focus on the analysis of the disconnected contributions using seven ensembles with simulation parameters given in Table I. By combining these with the connected contributions given in Eq. (2), we obtain final results for the flavor diagonal tensor charges.

\section{CONTROLLING EXCITED-STATE CONTAMINATION}

The first step in the analysis is to understand and remove the excited state contamination (ESC) in the disconnected contribution. A number of features stand out in the data shown in Fig. 1. First, for a given value of the sourcesink separation $\tau$, the data are much more noisy compared to the corresponding connected contribution analyzed in Ref. [13]. Second, within statistical uncertainties, there is no discernible variation with $\tau$. In fact, the data at the various values of $\tau$ overlap for both the light, $g_{T}^{l}$, and the strange, $g_{T}^{s}$, quark contributions; i.e., no ESC is apparent in either. Lastly, the magnitude, in most cases, is smaller than 0.01 , which is smaller than the statistical uncertainty in the connected contribution. Possible residual ESC is expected to be even smaller. The bottom line is, for the estimate on each ensemble, we take a simple average over the multiple $t$ and $\tau$ data shown in Fig. 1. These results for the bare charges, $g_{T}^{l}$ and $g_{T}^{s}$, are compiled together in Table II.

In Refs. [12,13], we raised the need for evaluating the uncertainty due to analyzing the connected and disconnected contributions separately to remove the ESC using the QCD spectral decomposition. For the tensor charges, this uncertainty is expected to be negligible for two reasons: The disconnected data show no evidence for ESC and we take the average over the various $\tau$ values; i.e., no fits using the spectral decomposition are made. Second, the magnitude of the contributions, $<0.01$, is smaller than the combined statistical errors. So, we assume that any residual uncertainty due to performing separate analyses will be even smaller.

\section{RENORMALIZATION OF THE OPERATORS}

Flavor diagonal light-quark operators, $\bar{q} \Gamma q$, can be written as a sum over isovector $(u-d)$ and isoscalar $(u+d)$ combinations which renormalize differently-isovector with $Z^{\text {isovector }}$ and isoscalar with $Z^{\text {isoscalar }}$. The difference between $Z^{\text {isovector }}$ and $Z^{\text {isoscalar }}$ for quark bilinear operators starts, in general, at two loops in perturbation theory. For the tensor operator, the two-loop terms are zero because the spin trace vanishes in the clover, HISQ, and thus clover-onHISQ formulations [14]. Also, for the twisted mass action, nonperturbative calculations show $Z_{T}^{\text {isovector }}=Z_{T}^{\text {isoscalar }}$ to within a percent $[15,16]$. We have not calculated $Z_{T}^{\text {isoscalar }}$ nonperturbatively for the clover-on-HISQ formulation, which has additional $O(a)$ chiral breaking versus the twisted mass action. In this work, we assume that the difference is smaller than the statistical errors. The isovector renormalization constants $Z_{T}^{\text {isovector }}$, calculated in the regularizationindependent symmetric momentum-subtraction scheme and converted to the $\overline{\mathrm{MS}}$ scheme at $2 \mathrm{GeV}$ using two-loop perturbation theory, are taken from Ref. [13] and used to renormalize the connected and disconnected contributions to $g_{T}^{u}, g_{T}^{d}$ and $g_{T}^{s}$ in two ways:

$$
\begin{aligned}
& \left.g_{T}^{l, s}\right|_{R 1}=g_{T} \times Z_{T}^{\text {isovector }}, \\
& \left.g_{T}^{l, s}\right|_{R 2}=\frac{g_{T}}{g_{V}^{u-d}} \times \frac{Z_{T}^{\text {isovector }}}{Z_{V}^{u-d}} .
\end{aligned}
$$

The conserved vector charge condition $g_{V}^{u-d} \times Z_{V}^{u-d}=1$ is implicit in the second definition. These two results for the renormalized disconnected contributions on each ensemble are also given in Table II. They are extrapolated separately to the continuum limit and $M_{\pi}=135 \mathrm{MeV}$, and the extrapolated results are given in Table III.

TABLE II. The values of $a$ and $M_{\pi}$ for the seven ensembles are given in columns 2 and 3. Results for the unrenormalized disconnected light and strange quark contributions, $g_{T}^{l, s}$, are given in columns 4 and 5 . They are obtained using a simple average over the data shown in Fig. 1 since no significant ESC is evident. Columns 2 and 3 give the lattice spacing of the HISQ ensembles and the valence $M_{\pi}$, as described in Ref. [13]. In columns 6-9, we give the renormalized charges $\left.g_{T}^{l, s}\right|_{R 1}$ and $\left.g_{T}^{l, s}\right|_{R 2}$ defined in Eq. (3). The isovector renormalization constant $Z_{T}^{\text {isovector }}$ is used in all cases as discussed in the text.

\begin{tabular}{lcccccccc}
\hline \hline Ensemble ID & $a(\mathrm{fm})$ & $M_{\pi}(\mathrm{MeV})$ & \multicolumn{1}{c}{$\left.g_{T}^{l}\right|_{\text {bare }}$} & \multicolumn{1}{c}{$\left.g_{T}^{s}\right|_{\text {bare }}$} & \multicolumn{1}{c}{$\left.g_{T}^{l}\right|_{R 1}$} & $\left.g_{T}^{s}\right|_{R 1}$ & $\left.g_{T}^{l}\right|_{R 2}$ & $\left.g_{T}^{s}\right|_{R 2}$ \\
\hline a15m310 & $0.151(2)$ & $320(5)$ & $-0.0057(16)$ & $-0.0015(8)$ & $-0.0054(15)$ & $-0.0014(8)$ & $-0.0054(15)$ & $-0.0014(8)$ \\
a12m310 & $0.121(1)$ & $310(3)$ & $-0.0086(14)$ & $-0.0023(9)$ & $-0.0081(14)$ & $-0.0021(8)$ & $-0.0084(14)$ & $-0.0022(9)$ \\
a12m220 & $0.118(1)$ & $228(2)$ & $-0.0063(24)$ & $-0.0018(14)$ & $-0.0059(23)$ & $-0.0017(13)$ & $-0.0061(23)$ & $-0.0017(14)$ \\
a09m310 & $0.089(1)$ & $313(3)$ & $-0.0057(10)$ & $-0.0016(9)$ & $-0.0056(10)$ & $-0.0016(9)$ & $-0.0058(10)$ & $-0.0017(9)$ \\
a09m220 & $0.087(1)$ & $226(2)$ & $-0.0070(21)$ & $-0.0016(9)$ & $-0.0069(21)$ & $-0.0016(9)$ & $-0.0071(21)$ & $-0.0016(9)$ \\
a09m130 & $0.087(1)$ & $138(1)$ & & $-0.0016(21)$ & & $-0.0016(21)$ & & $-0.0016(21)$ \\
a06m310 & $0.058(1)$ & $320(2)$ & $-0.0055(11)$ & $-0.0033(11)$ & $-0.0057(12)$ & $-0.0034(11)$ & $-0.0059(12)$ & $-0.0035(12)$ \\
\hline \hline
\end{tabular}


TABLE III. Results, in the $\overline{\mathrm{MS}}$ scheme at $2 \mathrm{GeV}$, for the renormalized disconnected contributions to the proton's tensor charges were obtained in the limit $a=0$ and $M_{\pi^{0}}=135 \mathrm{MeV}$ by performing a chiral-continuum extrapolation using Eq. (4). The $\chi^{2} / \mathrm{DOF}$ of the two fits and the results (labeled $R 1$ and $R 2$ ) for the renormalized charges defined in Eq. (3) are given along with the final results obtained by averaging $\left.g_{T}^{l, s}\right|_{R 1}$ and $\left.g_{T}^{l, s}\right|_{R 2}$ and taking the larger of the two errors.

\begin{tabular}{|c|c|c|c|c|c|c|c|c|c|c|}
\hline & \multicolumn{5}{|c|}{ Light } & \multicolumn{5}{|c|}{ Strange } \\
\hline & $\overline{\left.g_{T}^{l}\right|_{R 1}}$ & $\chi^{2} / \mathrm{DOF}$ & $\overline{\left.g_{T}^{l}\right|_{R 2}}$ & $\chi^{2} / \mathrm{DOF}$ & $g_{T}^{l}$ & $\overline{\left.g_{T}^{s}\right|_{R 1}}$ & $\chi^{2} / \mathrm{DOF}$ & $\overline{\left.g_{T}^{s}\right|_{R 2}}$ & $\chi^{2} / \mathrm{DOF}$ & $g_{T}^{S}$ \\
\hline$\overline{g_{T}^{\text {disc }}}$ & $-0.0062(33)$ & 0.85 & $-0.0066(33)$ & 0.91 & $-0.0064(33)$ & $-0.0026(15)$ & 0.31 & $-0.0027(16)$ & 0.29 & $-0.0027(16)$ \\
\hline
\end{tabular}

\section{THE CONTINUUM-CHIRAL EXTRAPOLATION}

The last step in the analysis is to evaluate the results at $M_{\pi^{0}}=135 \mathrm{MeV}$ and in the continuum and infinite volume limits, $a \rightarrow 0$ and $M_{\pi} L \rightarrow \infty$. Over the limited range of $M_{\pi} L$ spanned by our disconnected data, $3.9<M_{\pi} L<4.8$, finite-volume corrections were negligible in the connected contributions, as shown in Fig. 2 in Ref. [13]. We, therefore, assume possible finite-volume corrections can be neglected in the disconnected contributions and fit the renormalized data given in Table II keeping just the leading correction terms in $a$ and $M_{\pi}$ :

$$
g_{T}^{l, s}\left(a, M_{\pi}, L\right)=c_{1}+c_{2} a+c_{3} M_{\pi}^{2}+\cdots .
$$

The data with the renormalization method $R 2$ and the results of the fits are shown in Fig. 2. The dependence of both $g_{T}^{l}$ and $g_{T}^{s}$ on $M_{\pi}$ and $a$ is small and the extrapolated value is consistent with an average over the six (seven) points. Given this consistency between the average values and the results of the linear extrapolation using Eq. (4), and applying the Akaike Information Criteria [17] (see Table III for the $\chi^{2}$ /DOF of the fits), including additional higher order corrections to the chiral-continuum fit ansatz, Eq. (4), is not warranted.

We consider the errors from the fit reasonable as they are larger than those in most individual points and cover the total range of variation in the points. Since the difference between the extrapolated results given in Table III for the two ways of doing the renormalization is much smaller than these errors, for the final value we take the average of the two as summarized in Tables III and IV.
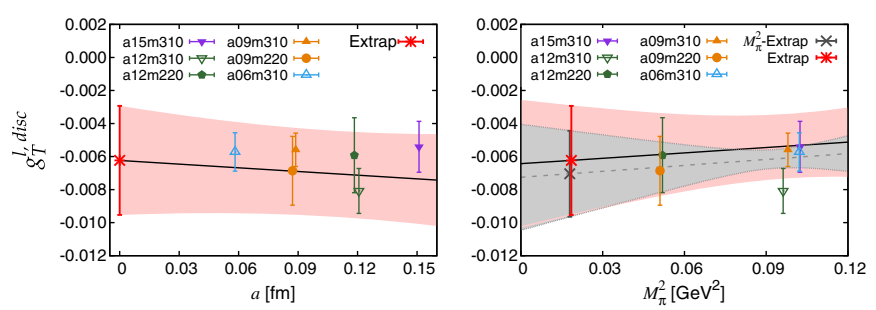

In the connected contributions to $g_{T}^{u}$ and $g_{T}^{d}$, analyzed in Ref. [13], a systematic uncertainty of 0.01 was assessed to account for residual uncertainty in the chiral-continuumfinite-volume fits made with only the leading order corrections. This 0.01 is quoted as the second error in the final results for $g_{T}^{u}$ and $g_{T}^{d}$ given in Table IV.

\section{COMPARISON WITH PREVIOUS WORK}

In Table IV, we show that results obtained by the ETMC Collaboration [15] using a single physical mass ensemble generated with two flavors of maximally twisted mass fermions with a clover term at $a=0.0938(4) \mathrm{fm}, M_{\pi}=$ 130.5(4) $\mathrm{MeV}$ and at much smaller $M_{\pi} L=2.98$ agree with our more complete analysis. Such consistency is expected if the differences due to the number of dynamical flavors, and possible discretization and finite-volume corrections in the ETMC'17 results, are small or cancel.

\section{IMPLICATIONS FOR NEUTRON ELECTRIC DIPOLE MOMENT}

The tensor charges for the neutron are, in the isospin symmetric limit, obtained from the proton charges by interchanging the light-quark labels, $u \leftrightarrow d$. Using the values given in Table IV and the experimental bound on the $\operatorname{nEDM}\left(d_{n} \leq 2.9 \times 10^{-26} e \mathrm{~cm}[18]\right)$, the relation

$$
d_{n}=d_{u}^{\gamma} g_{T}^{u}+d_{d}^{\gamma} g_{T}^{d}+d_{s}^{\gamma} g_{T}^{s},
$$

provides constraints on the $C P$-violating quark EDMs, $d_{q}^{\gamma}$, arising in beyond the Standard Model (BSM) theories, assuming that the quark EDM is the only $C P$-violating
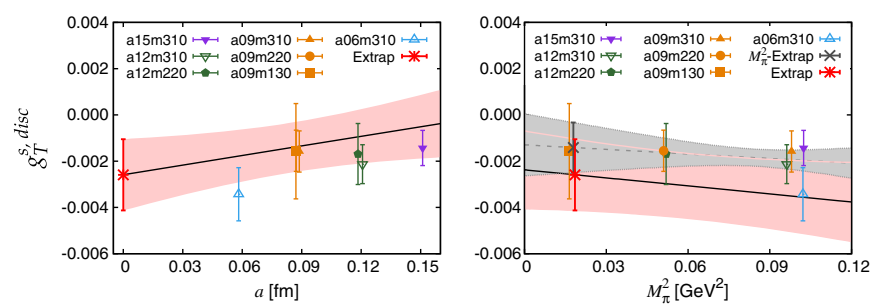

FIG. 2. The data, in the $\overline{\mathrm{MS}}$ scheme at $2 \mathrm{GeV}$, for the disconnected contribution $\left.g_{T}^{l \text {,disc }}\right|_{R 2}$ (left two panels) and $\left.g_{T}^{s, \text { disc }}\right|_{R 2}$ (right two panels) plotted versus $a$ and $M_{\pi}$. In each panel, the result at $a=0$ and $M_{\pi}=135 \mathrm{MeV}$, obtained using Eq. (4), is shown by the red star. The pink band is the fit shown versus $a\left(M_{\pi}\right)$, with the other variable set to its physical value. For comparison, the gray band between dotted lines shows a simpler linear fit versus only $M_{\pi}^{2}$, i.e., ignoring the dependence on $a$. 
TABLE IV. Final results, in the $\overline{\mathrm{MS}}$ scheme at $2 \mathrm{GeV}$, for the individual connected and disconnected contributions to the flavor diagonal tensor charges and their sum, labeled PNDME' 18 in the third row. The fourth row gives the ETMC results [15] for comparison. These were obtained from a single physical mass ensemble at $a=0.0938(4)$ and $M_{\pi}=130.5(4) \mathrm{MeV}$, i.e., without a continuum extrapolation and at small $M_{\pi} L=2.98$. Comparing PNDME'18 and PNDME'15 results [5] highlights the improvements realized with higher statistics and more ensembles; in particular, we now present results for light-quark disconnected contributions.

\begin{tabular}{lccc}
\hline \hline & \multicolumn{1}{c}{$g_{T}^{u}$} & $g_{T}^{d}$ & $g_{T}^{s}$ \\
\hline Connected & $0.790(27)$ & $-0.198(10)$ & \\
Disconnected & $-0.0064(33)$ & $-0.0064(33)$ & $-0.0027(16)$ \\
PNDME'18 & $0.784(28)(10)$ & $-0.204(11)(10)$ & $-0.0027(16)$ \\
ETMC'17 [15] & $0.782(21)$ & $-0.219(17)$ & $-0.00319(72)$ \\
PNDME'15 [5] & $0.774(66)$ & $-0.233(28)$ & $0.008(9)$ \\
\hline \hline
\end{tabular}

BSM operator. The bounds on $d_{q}^{\gamma}$ are shown in the left panel of Fig. 3. Of particular importance is the reduction in the error in $g_{T}^{s}$ compared to our previous result, $g_{T}^{s}=0.008(9)$, in Ref. [6]. The new result lets us bound $d_{s}^{\gamma}$. Conversely, the overall error in $d_{n}$ is reduced even if $d_{s}^{\gamma}$ is enhanced versus $d_{u}^{\gamma}$ by $m_{s} / m_{u} \approx 40$ as occurs in models in which the chirality flip is provided by the Standard Model Yukawa couplings.

In general, BSM theories generate a variety of $C P$-violating operators that all contribute to $d_{n}$ with relations analogous to Eq. (5). As discussed in Ref. [6], in the "split-supersymmetry (SUSY)" model [20-22], the fermion EDM operators provide the dominant BSM source of $C P$ violation. In Fig. 3 (right), we therefore update the contour plots for $d_{n} / d_{e}$ in the gaugino $\left(M_{2}\right)$ and Higgsino $(\mu)$ mass parameter plane with the range $500 \mathrm{GeV}$ to $10 \mathrm{TeV}$. For this analysis, we have followed Ref. [23] and set $\tan \beta=1$.

Thanks to the greatly reduced uncertainty in the tensor charges (factor of $\approx 6$ for $g_{T}^{s}$ and $\approx 2$ for $g_{T}^{l}$ ), the ratio $d_{n} / d_{e}$ is much more precisely known in terms of SUSY mass parameters. This allows for stringent tests of the splitSUSY scenario with gaugino mass unification [20-22]. In particular, our results and the experimental bound $d_{e}<1.1 \times 10^{-29} e \mathrm{~cm}[19,24]$ imply the split-SUSY upper bound $d_{n}<4.1 \times 10^{-29} e \mathrm{~cm}$. This limit is falsifiable by the next-generation nEDM experiments. Constraints on split SUSY from LHC searches predicated on gluino decays rule out the region below about a TeV in the $\left\{\mu, M_{2}\right\}$ plane [25], whereas, assuming a maximal $C P$-violating phase $(\sin \phi=1)$, EDMs currently probe scales considerably higher than LHC's reach.

\section{CONCLUSIONS}

We present results for the flavor diagonal tensor charges $g_{T}^{u}, g_{T}^{d}$ and $g_{T}^{s}$, with control over all the systematics for both the connected and the disconnected contributions. The light disconnected contributions, which were neglected in the PNDME'15 publication [5], are small and show little variation versus the lattice spacing or the pion mass. The errors in the individual connected and the disconnected contributions on each ensemble have been significantly reduced due to the high statistics. The final results, given in Table IV, were obtained using a controlled chiralcontinuum fit to data on multiple ensembles that cover a
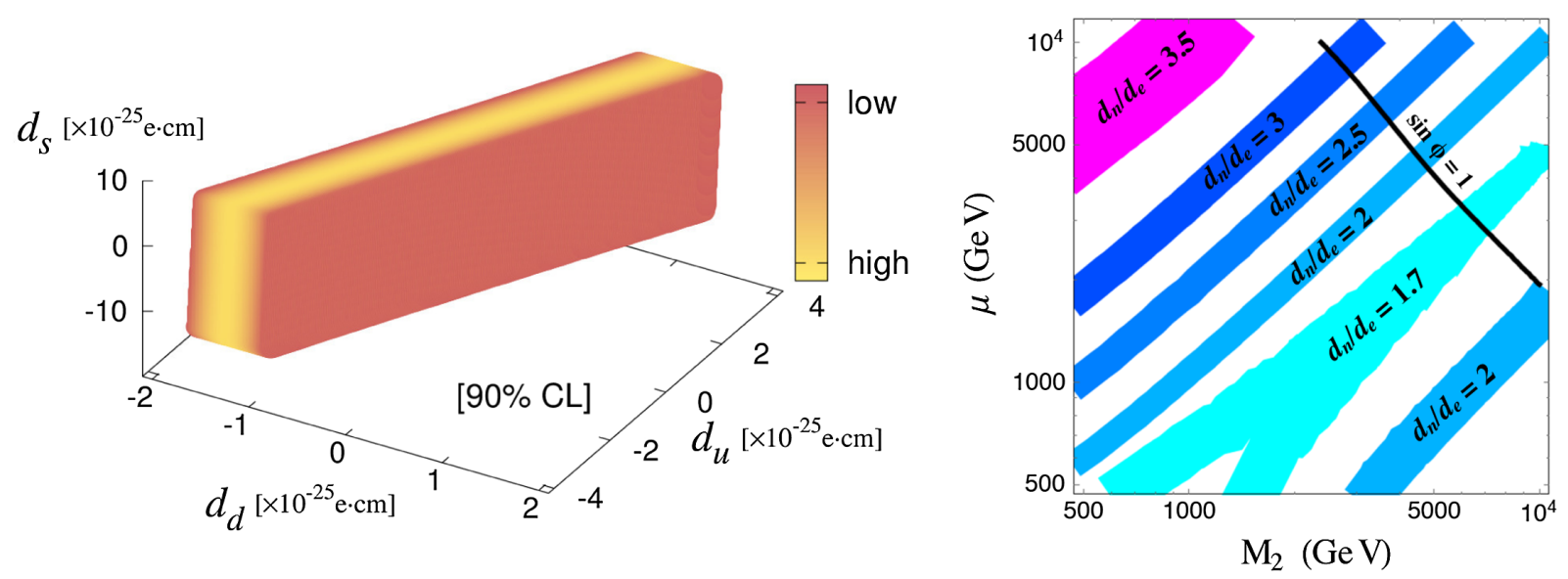

FIG. 3. (Left) Constraints on the BSM couplings of the $C P$-violating quark EDM operator using the current experimental bound on the $\operatorname{nEDM}\left(2.9 \times 10^{-26} e \mathrm{~cm}[18]\right)$ and assuming that only these couplings contribute. The strongest constraint is a strip in $d_{u}$ and $d_{d}$, i.e., representing the thickness of the slab, with high (low) corresponding to a $p$ value $=1(0.1)$. (Right) Regions in the $M_{2}-\mu$ plane corresponding to various values of $d_{n} / d_{e}$ in split SUSY, obtained by varying $g_{T}^{u, d, s}$ within our estimated uncertainties. In the bands of constant $d_{n} / d_{e}$, the values of both $d_{n}$ and $d_{e}$ decrease as $\mu$ and $M_{2}$ increase. Using $d_{e} \leq 1.1 \times 10^{-29}$ e cm [19] and assuming maximal $C P$ violation $(\sin \phi=1)$, the allowed region lies above the solid black line. For $\mu, M_{2}>500 \mathrm{GeV}$, maximizing the ratio $d_{n} / d_{e}$ along this line gives the upper bound $d_{n}<4.1 \times 10^{-29} e \mathrm{~cm}$ at $d_{n} / d_{e}=3.71$. 
sufficiently large range in lattice spacing and pion mass. The reduced errors have allowed us to tighten the constraints on the quark EDM couplings and on the ratio $d_{n} / d_{e}$ in the split-SUSY scenario with gaugino mass unification [20-22] as shown in Fig. 3.

\section{ACKNOWLEDGMENTS}

We thank the MILC Collaboration for providing the $(2+1+1)$-flavor HISQ lattices used in our calculations. The calculations used the Chroma software suite [26]. Simulations were carried out on computer facilities of (i) the National Energy Research Scientific Computing Center, a DOE Office of Science User Facility supported by the Office of Science of the U.S. Department of Energy under Contract No. DE-AC02-05CH11231; (ii) the Oak
Ridge Leadership Computing Facility at the Oak Ridge National Laboratory, which is supported by the Office of Science of the U.S. Department of Energy under Contract No. DE-AC05-00OR22725; (iii) the USQCD Collaboration, which are funded by the Office of Science of the U.S. Department of Energy; and (iv) Institutional Computing at Los Alamos National Laboratory. T. B. and R. G. were partly supported by the U.S. Department of Energy, Office of Science, Office of High Energy Physics under Contract No. DE-AC52-06NA25396. T. B., V. C., R. G., Y-C. J. and B. Y. were partly supported by the LANL LDRD program. The work of H.-W. L. is supported by the U.S. National Science Foundation under Grant No. PHY 1653405 "CAREER: Constraining Parton Distribution Functions for New-Physics Searches."
[1] H.-W. Lin, W. Melnitchouk, A. Prokudin, N. Sato, and H. Shows, Phys. Rev. Lett. 120, 152502 (2018).

[2] M. Radici and A. Bacchetta, Phys. Rev. Lett. 120, 192001 (2018).

[3] Z. Ye, N. Sato, K. Allada, T. Liu, J.-P. Chen, H. Gao, Z.-B. Kang, A. Prokudin, P. Sun, and F. Yuan, Phys. Lett. B 767, 91 (2017).

[4] F. Bishara, J. Brod, B. Grinstein, and J. Zupan, J. High Energy Phys. 11 (2017) 059.

[5] T. Bhattacharya, V. Cirigliano, S. Cohen, R. Gupta, A. Joseph, H.-W. Lin, and B. Yoon (PNDME Collaboration), Phys. Rev. D 92, 094511 (2015).

[6] T. Bhattacharya, V. Cirigliano, R. Gupta, H.-W. Lin, and B. Yoon, Phys. Rev. Lett. 115, 212002 (2015).

[7] E. Follana, Q. Mason, C. Davies, K. Hornbostel, G. P. Lepage, J. Shigemitsu, H. Trottier, and K. Wong (HPQCD and UKQCD Collaborations), Phys. Rev. D 75, 054502 (2007).

[8] A. Bazavov et al. (MILC Collaboration), Phys. Rev. D 87, 054505 (2013).

[9] T. Bhattacharya, V. Cirigliano, S. Cohen, R. Gupta, H.-W. Lin, and B. Yoon, Phys. Rev. D 94, 054508 (2016).

[10] B. Yoon et al., Phys. Rev. D 93, 114506 (2016).

[11] R. Gupta, Y.-C. Jang, H.-W. Lin, B. Yoon, and T. Bhattacharya, Phys. Rev. D 96, 114503 (2017).

[12] H.-W. Lin, R. Gupta, B. Yoon, Y.-C. Jang, and T. Bhattacharya, arXiv:1806.10604.
[13] R. Gupta, Y.-C. Jang, B. Yoon, H.-W. Lin, V. Cirigliano, and T. Bhattacharya, Phys. Rev. D 98, 034503 (2018).

[14] M. Constantinou, M. Hadjiantonis, H. Panagopoulos, and G. Spanoudes, Phys. Rev. D 94, 114513 (2016).

[15] C. Alexandrou et al., Phys. Rev. D 95, 114514 (2017); 96, 099906(E) (2017).

[16] C. Alexandrou, M. Constantinou, K. Hadjiyiannakou, K. Jansen, C. Kallidonis, G. Koutsou, A. Vaquero Avils-Casco, and C. Wiese, Phys. Rev. Lett. 119, 142002 (2017).

[17] H. Akaike, IEEE Trans. Autom. Control 19, 716 (1974).

[18] C. Baker, D. Doyle, P. Geltenbort, K. Green, M. van der Grinten et al., Phys. Rev. Lett. 97, 131801 (2006).

[19] V. Andreev et al. (ACME Collaboration), Nature (London) 562, 355 (2018).

[20] N. Arkani-Hamed and S. Dimopoulos, J. High Energy Phys. 06 (2005) 073.

[21] G. Giudice and A. Romanino, Nucl. Phys. B699, 65 (2004).

[22] N. Arkani-Hamed, S. Dimopoulos, G. Giudice, and A. Romanino, Nucl. Phys. B709, 3 (2005).

[23] G. Giudice and A. Romanino, Phys. Lett. B 634, 307 (2006).

[24] J. Baron et al. (ACME Collaboration), Science 343, 269 (2014).

[25] A. M. Sirunyan et al. (CMS Collaboration), J. High Energy Phys. 05 (2018) 025.

[26] R. G. Edwards and B. Joo (SciDAC, LHPC, and UKQCD Collaborations), Nucl. Phys. B, Proc. Suppl. 140, 832 (2005). 